\title{
Modular statistics for subgraph counts in sparse random graphs
}

\author{
Bobby DeMarco* \\ rvdemarco@gmail.com
}

\author{
Amanda Redlich ${ }^{\ddagger}$ \\ Department of Mathematics \\ Bowdoin College \\ Brunswick, ME, U.S.A. \\ aredlich@bowdoin.edu
}

Submitted: Feb 6, 2014; Accepted: Jan 28, 2015; Published: Feb 16, 2015

Mathematics Subject Classifications: 05C80, 03C13

\begin{abstract}
Answering a question of Kolaitis and Kopparty, we show that, for given integer $q>1$ and pairwise nonisomorphic connected graphs $G_{1}, \ldots, G_{k}$, if $p=p(n)$ is such that $\operatorname{Pr}\left(G_{n, p} \supseteq G_{i}\right) \rightarrow 1 \forall i$, then, with $\xi_{i}$ the number of copies of $G_{i}$ in $G_{n, p}$, $\left(\xi_{1}, \ldots, \xi_{k}\right)$ is asymptotically uniformly distributed on $\mathbf{Z}_{q}^{k}$.
\end{abstract}

\section{Introduction}

For graphs $G, H$ write $N(G, H)$ for the number of unlabeled copies of $H$ in $G$ (e.g. $\left.N\left(K_{r}, K_{s}\right)=\left(\begin{array}{l}r \\ s\end{array}\right)\right)$. We use both $G_{n, p}$ and $G(n, p)$ for the ordinary ("binomial" or "ErdősRényi") random graph.

We are interested here in extending to nonconstant $p$ the following beautiful result of Kolaitis and Kopparty [4].

Theorem 1. Fix an integer $q>1, p \in(0,1)$ and pairwise nonisomorphic connected graphs $G_{1}, \ldots, G_{k}$, each with at least two vertices, and let $\xi_{i}$ be $N\left(G_{n, p}, G_{i}\right)(\bmod q)$. Then the distribution of $\xi=\left(\xi_{1}, \ldots, \xi_{k}\right)$ is $e^{-\Omega(n)}$-close to uniform on $\mathbf{Z}_{q}^{k}$. In particular, for each $a \in \mathbf{Z}_{q}^{k}, \operatorname{Pr}(\xi=a) \rightarrow q^{-k}$ as $n \rightarrow \infty$.

(Recall two distributions are $\varepsilon$-close if their statistical (a.k.a. variation) distance is at most $\varepsilon$.) Essentially, this theorem states that for constants $p$ and $q$, subgraphs of $G(n, p)$ are uniformly distributed modulo $q$.

${ }^{*}$ Supported by NSF grant DMS1201337.

${ }^{\dagger}$ Supported by NSF grant DMS1201337.

¥Supported by NSF Award No. 1004382. 
Theorem 1 was motivated by an application to 0-1 laws for first order logic with a parity quantifier or, more generally, a quantifier that allows counting modulo $q$; see Section 3 for a little more on this.

A natural question raised in [4] (and communicated to the authors by S.K.) asks, to what extent does Theorem 1 remain true if $p$ is allowed to tend to zero as $n$ grows, e.g. if $p=n^{-\alpha}$ for some fixed $\alpha>0$ ? Our purpose here is to answer this question.

We need a little notation. For a graph $H=(V, E)$, set $v_{H}=|V|, e_{H}=|H|:=|E|$, $\rho(H)=e_{H} / v_{H}$ and $m(H)=\max \left\{\rho\left(H^{\prime}\right): H^{\prime} \subseteq H, v_{H^{\prime}}>0\right\}$. Recall (see e.g. [2]) that $n^{-1 / m(H)}$ is a threshold function for containment of $H$; that is, the probability that $G_{n, p}$ $(p=p(n))$ contains a copy of $H$ tends to 0 if $p n^{1 / m(H)} \rightarrow 0$ and to 1 if $p n^{1 / m(H)} \rightarrow \infty$. Given a collection $\mathcal{G}$ of graphs, set $m(\mathcal{G})=\max \{m(G): G \in \mathcal{G}\}, p_{\mathcal{G}}(n)=n^{-1 / m(\mathcal{G})}$ and

$$
\Phi_{\mathcal{G}}(n, p)=\min _{G \in \mathcal{G}} \min \left\{n^{v_{H}} p^{e_{H}}: H \subseteq G, v_{H}>0\right\} .
$$

Theorem 2. Let $q, G_{1}, \ldots, G_{k}$ and $\xi=\left(\xi_{1}, \ldots, \xi_{k}\right)$ be as in Theorem 1 and $\mathcal{G}=$ $\left\{G_{1}, \ldots, G_{k}\right\}$. If $p=\omega\left(p_{\mathcal{G}}(n)\right)$, then the distribution of $\xi$ is $\exp \left[-\Omega\left(\Phi_{\mathcal{G}}(n, p)\right)\right]$-close to uniform on $\mathbf{Z}_{q}^{k}$.

(Of course the constant in the exponent depends on $q$ and $\mathcal{G}$.)

Suppose e.g. that $q=k=2, G_{1}=K_{3}$, and $G_{2}=K_{4}$. Then $m(\mathcal{G})=m\left(G_{2}\right)=3 / 2$ $\left(m\left(G_{1}\right)=1\right)$ and $p_{\mathcal{G}}(n)=n^{-2 / 3}$, so the theorem says that, asymptotically speaking, the parities of the numbers of copies of $K_{3}$ and $K_{4}$ are independent with each equally likely to be even or odd, provided $p=\omega\left(n^{-2 / 3}\right)$.

For the special case $\mathcal{G}=\left\{K_{3}\right\}$, a somewhat weaker version of Theorem 2 -with $\exp \left[-\Omega\left(\Phi_{\mathcal{G}}(n, p)\right)\right]$ replaced by something polynomial in $n$ and $p$-has been shown by Noga Alon [3].

We should also note here an immediate consequence of Theorem 2, which again answers a question from [4].

Corollary 3. Let $q, \mathcal{G}$ be as in Theorem 1, fix a positive irrational $\alpha$, and let $I=\{i \in$ $\left.[k]: m\left(G_{i}\right)<\alpha^{-1}\right\}$ and $J=[k] \backslash I$. Then for $p=n^{-\alpha}$ and $a \in \mathbf{Z}_{q}^{k}$ (and $\xi$ as in Theorem 1),

$$
\operatorname{Pr}(\xi=a) \rightarrow \begin{cases}q^{-|I|} & \text { if } a_{j}=0 \forall j \in J, \\ 0 & \text { otherwise. }\end{cases}
$$

This is of interest partly for its possible relevance to proving a modular convergence law (again see Section 3) for $p=n^{-\alpha}$ with $\alpha$ irrational (cf. [5, Theorem 6], which says that for such $p$ a 0-1 law holds for any first order property); but we also have, again from [4]: "Even the behavior of subgraph frequencies mod 2 in this setting [i.e. with $p$ as in Corollary 3] seems quite intriguing."

The proof of Theorem 2, given in the next section, is similar to that of Theorem 1 in [4]. In truth, we just add one little idea to the machinery of [4]; nonetheless, as the proof answers a rather basic question, and was apparently not quite trivial to find, it seems worth recording. 


\section{Proof}

We will need the following two facts, the first of which, from [4], generalizes a result of Babai, Nisan and Szegedy [1].

Lemma 4. Let $q>1$ and $d>0$ be integers and $p \in(0,1)$. Let $\mathcal{F} \subseteq 2^{[m]}$ and let $Q\left(z_{1}, \ldots z_{m}\right) \in \mathbf{Z}_{q}\left[z_{1}, \ldots z_{m}\right]$ be a polynomial of the form

$$
\sum_{S \in \mathcal{F}} a_{S} \prod_{i \in S} z_{i}+Q^{\prime}\left(z_{1}, \ldots z_{m}\right)
$$

where $\operatorname{deg}\left(Q^{\prime}\right)<d$. Suppose there is some $\mathcal{E}=\left\{E_{1}, \ldots E_{r}\right\} \subseteq \mathcal{F}$ such that

- $\left|E_{j}\right|=d$ for all $j$,

- $a_{E_{j}} \neq 0$ for all $j$,

- $E_{j} \cap E_{j^{\prime}}=\emptyset$ for all $j \neq j^{\prime}$, and

- for each $S \in \mathcal{F} \backslash \mathcal{E},\left|S \cap\left(\cup_{j} E_{j}\right)\right|<d$.

Let $\mathbf{z}=\left(\mathbf{z}_{1}, \ldots \mathbf{z}_{m}\right) \in \mathbf{Z}_{q}^{m}$ be the random variable where, independently for each $i, \operatorname{Pr}\left(\mathbf{z}_{i}=\right.$ $1)=p$ and $\operatorname{Pr}\left(\mathbf{z}_{i}=0\right)=1-p$. Then for $\omega \in \mathbf{C}$ a primitive $q^{\text {th }}$-root of unity,

$$
\left|\mathrm{E}\left[\omega^{Q(\mathbf{z})}\right]\right| \leqslant 2^{-\Omega(r)} .
$$

(We again observe that the implied constant in the $\Omega(r)$ term depends on $q, p$ and $d$.)

Lemma 5 ("Vazirani XOR Lemma"). Let $q>1$ be an integer and $\omega \in \mathbf{C}$ a primitive $q^{\text {th }}$-root of unity. Let $\xi=\left(\xi_{1}, \ldots, \xi_{l}\right)$ be a random variable taking values in $\mathbf{Z}_{q}^{l}$. Suppose that for every nonzero $c \in \mathbf{Z}_{q}^{l}$,

$$
\left|\mathrm{E}\left[\omega^{\sum c_{i} \xi_{i}}\right]\right| \leqslant \epsilon
$$

Then the distribution of $\xi$ is $\left(q^{l} \epsilon\right)$-close to uniform on $\mathbf{Z}_{q}^{l}$.

Proof of Theorem 2. Letting $e$ run over edges of $K_{n}$, the argument of [4] expresses each $\sum c_{i} \xi_{i}$ in the natural way as a polynomial in the indicators $\mathbf{z}_{e}:=\mathbf{1}_{\{e \in G(n, p)\}}\left(e \in E\left(K_{n}\right)\right)$ namely,

$$
\sum_{i} c_{i} \xi_{i}=\sum_{i} c_{i} \sum\left\{\prod_{e \in H} \mathbf{z}_{e}: G_{i} \cong H \subseteq K_{n}\right\}
$$

-and for the $\mathcal{E}$ of Lemma 4 uses $\Omega(n)$ vertex-disjoint copies of some largest $G_{i}$ among those with $c_{i} \neq 0$. The problem with this in the present situation is the (hidden) dependence of the bound in (1) on $p$.

We get around this difficulty by choosing our random graph in two steps, so that when we come to apply Lemma 4 we are back to constant $p$. For simplicity we now write $\Phi$ for $\Phi_{\mathcal{G}}(n, p), \mathbf{G}^{\prime}$ for $G(n, 2 p)$ and $\mathbf{G}$ for the random subgraph of $\mathbf{G}^{\prime}$ in which each edge 
is present, independently of other choices, with probability $1 / 2$; in particular, our $\xi_{i}$ 's are functions of $\mathbf{G}(=G(n, p))$.

Given $\mathbf{G}^{\prime}$, we will apply Lemma 4 with variables $\mathbf{z}_{e}=\mathbf{1}_{\{e \in \mathbf{G}\}}\left(e \in \mathbf{G}^{\prime}\right), \mathcal{F}$ the collection of copies of $G_{1}, \ldots, G_{k}$ in $\mathbf{G}^{\prime}$, and $\mathcal{E} \subseteq \mathcal{F}$ a large collection of vertex-disjoint copies of an appropriate $G_{i}$; so first of all we need existence of such an $\mathcal{E}$. For a given $\varepsilon$, let $\mathcal{D}=\mathcal{D}_{\varepsilon}$ be the event that $\mathbf{G}^{\prime}$ contains, for each $i$, a collection of $r:=\varepsilon \Phi$ vertex-disjoint copies of $G_{i}$.

Proposition 6. There is a fixed $\varepsilon>0$ (depending on $\mathcal{G}$ ) for which

$$
\operatorname{Pr}(\overline{\mathcal{D}})<\exp [-\Omega(\Phi)]
$$

Proof.

Though we don't know a reference, this is presumably not new and the ideas needed to prove it may all be found in [2];

so we just indicate what's involved.

Fix $i \in[k]$ and write $H$ for $G_{i}$. Let $Y$ be the maximum size of a collection of disjoint copies of $H$ in $\mathbf{G}^{\prime}$. It is enough to show that the (more properly, "a") median of $Y$ is $\Omega(\Phi)$; (2) then follows via an inequality of Talagrand ([7] or [2, Theorem 2.29]) as in the argument for the edge-disjoint analogue of Proposition 6 given on page 77 of [2]. (In our case Talagrand's inequality says that for a median $m$ of $Y$ and $t>0$, $\operatorname{Pr}(Y \leqslant m-t) \leqslant 2 \exp \left[-t^{2} /(4 \psi(m))\right]$, where $\left.\psi(r)=r|H|.\right)$

For a lower bound on the median of $Y$, write $X$ for the number of copies of $H$ (in $\mathbf{G}^{\prime}$ ) and $Z$ for the number of (unordered) pairs of non-disjoint copies. Then:

(i) $\mathrm{E}(X)=\Omega(\Phi)$ (this is immediate from the definitions);

(ii) w.h.p. $X>(1-o(1)) \mathrm{E} X$ (a basic application of the 2nd moment method; see [2, Remark 3.7]);

(iii) $\mathrm{E} Z<c \mathrm{E}^{2} X / \Phi$ for a suitable fixed $c$ (a straightforward calculation using the definition of $\Phi)$, so with probability at least $3 / 4, Z<4 c \mathrm{E}^{2} X / \Phi$;

(iv) by Turán's Theorem (applied to the graph with vertices the copies of $H$, edges the non-disjoint pairs and (therefore) independence number $Y$; cf. [2, Eq. (3.21)]), $Y \geqslant X^{2} /(X+2 Z) ;$ and thus

(v) with probability at least $3 / 4-o(1)$,

$$
Y>\frac{(1-o(1)) \mathrm{E}^{2} X}{\mathrm{E} X+8 c \mathrm{E}^{2} X / \Phi}=\Omega(\Phi)
$$

(where the first inequality uses the fact that $x^{2} /(x+2 z)$ is increasing in $x$ for $x, z>0$ ).

In view of Proposition 6 it is enough to show that for any $G^{\prime}$ satisfying $\mathcal{D}$, the conditional distribution of $\xi$ given $\left\{\mathbf{G}^{\prime}=G^{\prime}\right\}$ is $\exp [-\Omega(\Phi)]$-close to uniform on $\mathbf{Z}_{q}^{k}$. Given such a $G^{\prime}$ and $\underline{0} \neq c \in \mathbf{Z}_{q}^{k}$, take $\mathcal{F}_{i}$ to consist of all copies of $G_{i}$ in $G^{\prime}(i \in[k])$ and $\mathcal{F}=\cup\left\{\mathcal{F}_{i}\right.$ : 
$\left.c_{i} \neq 0\right\}$. Fix, in addition, some $i_{0} \in[k]$ with $c_{i_{0}} \neq 0$ and $\left|G_{i_{0}}\right|=\max \left\{\left|G_{i}\right|: c_{i} \neq 0\right\}=: d$, and some $\mathcal{E}=\left\{E_{1}, \ldots, E_{r}\right\} \subseteq \mathcal{F}_{i_{0}}$, with the $E_{i}$ 's vertex-disjoint.

We have

$$
\sum_{i \in[k]} c_{i} \xi_{i}=\sum_{i \in[k]} c_{i} \sum_{H \in \mathcal{F}_{i}} \prod_{e \in H} \mathbf{z}_{e}=: Q(\mathbf{z})
$$

where $\mathbf{z}_{e}=\mathbf{1}_{\{e \in \mathbf{G}\}}$ for $e \in G^{\prime}$. We then need to say that $Q, \mathcal{F}$ and $\mathcal{E}$ (with $q, d$ and $p=1 / 2$ ) satisfy the requirements of Lemma 4 . But the first three of these are immediate and the fourth follows from the connectivity of the $G_{i}$ 's: for $H \in \mathcal{F} \backslash \mathcal{E}$, if $V(H) \nsubseteq$ $V\left(E_{i}\right) \forall i$, then (since $H$ is connected and the $E_{i}$ 's are vertex-disjoint) $H \nsubseteq \cup E_{i}$, whence $\left|H \cap\left(\cup E_{i}\right)\right|<|H| \leqslant d$; otherwise we have $V(H) \subseteq V\left(E_{j}\right)$ for some $j$ and, since $H \neq E_{j}$, $\left|H \cap\left(\cup E_{i}\right)\right|=\left|H \cap E_{j}\right|<\left|E_{i}\right|=d$. Thus Lemma 4 applies, yielding

$$
\left|\mathrm{E} \omega^{Q(z)}\right| \leqslant \exp [-\Omega(\Phi)]
$$

and then (since this was for any $c \neq \underline{0}$ ) Lemma 5 says that, as desired, the conditional distribution of $\xi$ given $\left\{\mathbf{G}^{\prime}=G^{\prime}\right\}$ is $\exp [-\Omega(\Phi)]$-close to uniform on $\mathbf{Z}_{q}^{k}$.

\section{Discussion}

As mentioned earlier, Theorem 1 is a key ingredient in the proof of the Kolaitis-Kopparty "modular convergence law" for first order logic with a parity quantifier, or, more generally, a quantifier that allows counting $\bmod q$. This law says, briefly, that, for fixed $p$ and $n \rightarrow \infty$, the probability of a given sentence in the system under consideration tends to a limit that depends only on the congruence class of $n \bmod q$. (See also [6] for an in-depth discussion of $0-1$ laws for random graphs.)

As suggested in [4], it would be interesting to understand to what extent such a law holds in the sparse setting. Theorem 2 gets about half way to this goal (for $p$ in its range); but the other half-an assertion like Theorem 2.3 of [4] to the effect that all relevant information is contained in the subgraph frequencies - seems to require something new, since the quantifier elimination process underlying that step depends critically on properties of $G(n, p)$ that hold for constant $p$ but fail when $p$ tends to zero.

In closing we just mention that it would be interesting to find a proof of Theorem 2 that proceeds from first principles and does not depend on the "generalized inner product" polynomials underlying Lemma 4.

\section{Acknowledgement}

We would like to thank Swastik Kopparty for telling us the problem and for helpful conversations on the material of Section 3. 


\section{References}

[1] L. Babai, N. Nisan and M. Szegedy, Multiparty protocols and logspace-hard pseudorandom sequences, in Proc. 21st ACM Symposium on the Theory of Computing, 1989.

[2] S. Janson, T. Łuczak and A. Ruciński, Random Graphs, Wiley-Interscience, 2000.

[3] S. Kopparty, personal communication.

[4] P. G. Kolaitis and S. Kopparty, Random graphs and the parity quantifier, J. ACM, vol. 60 (5) Article 37, 2013.

[5] S. Shelah and J. Spencer, Zero-one laws for sparse random graphs, J. Amer. Math. Soc. 1 (1988), 97-115.

[6] J. Spencer, The Strange Logic of Random Graphs, Springer, 2001

[7] M. Talagrand, Concentration of measure and isoperimetric inequalities in product spaces, Inst. Hautes Études Sci. Publ. Math. 81 (1995), 73-205. 